\title{
ESTUDIO COMPARATIVO DEL ZOOPLANCTON EN TRES ULLALES DEL PARQUE NATURAL DE LA ALBUFERA DE VALENCIA
}

\author{
M. T. Alonsoy M. R. Miracle \\ Depto. Ecologia.Fac. Ciències Biològiques. Universitat de Valencia.46100 Burjassot. Valencia.
}

Palabras clave:Zooplankton, spring pools.

\begin{abstract}
COMPARATIVE STUDY OF ZOOPLANKTON IN THREE SPRING POOLS OF THE ALBUFERA OF VALENCIA NATURAL PARK
\end{abstract}

The annual cycle of zooplankton in three spring pools of the same marsh area has been studied. They are small, shallow pools fed by subterranean water. Eachone of the spring pools has anunique zooplanktoncommunity, which is different again from that in the large lagoon, "Albufera", which gives the name to the marsh area. The optical impression on the degree of degradation of the pools corresponds to the species composition expected. In the more contaminated pool, "Romaní", a total number of 32 species was found, all of them cosmopolitan, wide-spread species. The plankton of this pool, except in May, was dominated by rotifers. However in the other two pools geographically and environmentaly restricted species were still found and in them copepods were much more important than in "Romani".In one of them, "Gros", rare species of subterraneandistributionsuchas Eucyclopsgraeteri, Phyllognathopys viguiere were present and the restricted Mycrocyclops rubellus major was the characteristic cyclopoid. In this pool 28 species were found. Finally the third pool, "Baldovina" had 43 species. Its exclusive cyclopoid was the rare Metacyclops planus. It is a pool slightly more saline than the others, so the above mentioned cyclopoid plus the harpacticoids Horsiellabrevicornis and Cletocamptus retrogressus occurred in it and not in the other pools. Alsoabig population of the restricted Dunhevediacrassa was registered in this pool together with populations of rotifers of the uncommongenus, Asplanchnopus, (A.multiceps and A. hyalinus) and Euchlanis dapidula.

Great differences inzooplankton populationdynamics between the three pools were also very apparent. Maxima occurred at different times of the year, mid and late spring and mid-summer, earliest in the most degradated pool and latest in the least degradated pool. The pols can be considered as refuge areas for restricted species.

\section{INTRODUCCIÓN}

Los ullales son surgencias que originan pequeñas charcas de agua semiestancada. En la zona Sur de la marjal de la Albufera de Valencia seencuentran vanos de estos reductos, que undía fueron parte integrante de la antigua Albufera, pero que en la actualidad, debido al proceso de aterramiento, han quedado entre campos de na- ranjos, como el ullal del Romaní o entre campos de arroz como los ullales Gros y de la Baldovina. Son ecosistemasque se mantienen bastante establesa lo largodel año en lo que se refiere a temperatura, sales minerales, $\mathrm{pH}$ y $\mathrm{O}$,. Sin embargo, la concentración de nutnentes es bastante variable, probablemente debida al abonado de los campos 
adyacentes. El ullal del Romaní presenta altas concentraciones de nitratos y es el que está más degradado y contaminado. Por otro lado el de la Baldovina, que es el mejor conservado, presenta una salinidad entre el 1 y el $2 \%$, ligeramente mayor y más variable que la de los otros dos ullales, que se mantienen entre el 0,5 y el $1 \%$. Finalmente el ullal Gros se caracteriza por la poca transparencia de sus aguas, debido a una gran densidad de partículas de carbonato cálcicoen suspensión.

En este artículo se presentan los resultados sobre las comunidades zooplanctónicasque pueblan tres ullalesdel Parque Natural de la Albufera de Valencia. Su objetivo es analizar la composición específica y distribución estacional del zooplancton de este tipo de ambientes, que debido a su régimen ligado al sistema freático, son aguas mucho menos contaminadas y manipuladas que las de la marjal circundante.

\section{MATERIAL Y MÉTODOS}

Se han estudiado tres ullalessituados entre los términos del Romaní y Sueca: (1) El ullal del Romaní (cuyas coordenadas geográficasson 39" $17^{\prime} \mathrm{N}$ y $0^{\circ} 23^{\prime} \mathrm{W}$ ) es de forma alargada, con una longitud de $64 \mathrm{~m}$ y $4.5 \mathrm{~m}$ de anchura mínima y 10 m de máxima. (2) El ullal Gros, de Albalat de la Ribera ( $39^{\circ} 13^{\prime} \mathrm{N}$ y $0^{\circ} 22^{\prime} \mathrm{W}$ ) tiene forma ovalada, siendo sus diámetros 57 x 35 m. (3) El ullal de la Baldovina, situado junto a la Muntanyeta dels Sants $\left(39^{\circ} 14^{\prime} \mathrm{N}\right.$ y $\left.0^{\circ} 18^{\prime} \mathrm{W}\right)$ es de forma redondeada, siendo su diámetro medio aproximado de 47 $\mathrm{m}$ y su profundiaad máxima de $2.8 \mathrm{~m}$.

Los ullales se muestrearon mensualmente durante un ciclo anual comprendido entre el $4 \mathrm{de}$ febrero de 1986 y el 24 de febrero de 1987; recogiéndose un total de 12 muestras de zooplancton en cada ullal, en un mismo punto de muestreo establecidocerca de la orilla. Las muestras fueron recogidas con una botella Ruttner de 2.6 litros de capacidad, filtrándoseel contenido con una malla de $50 \mathrm{~m}$. Las muestras filtradas eran seguidamente preservadas en formol al 5\%. Posteriormente se contaban los crustáceos y rotiferos con una magnificación de 100 ó 200 aumentos, mediante un microscopioinvertido.

\section{RESULTADOS}

Cada uno de los ullales presenta una comunidad zooplanctónica propia y particular que es además diferente de la que se mantiene en la Albufera de Valencia.La Albufera esactualmente un lago hipertrófico (Serra et al., 1984, Miracle et al.,1984)cuya fauna está muy empobrecida (Oltra y Miracle, 1987). Por constituir reductos de pequeño volumen, en el plancton de los ullales se encuentran una serie de especies de distribución litoral-bentónica en otros sistemas acuáticos de mayores dimensiones.

\section{Crustáceos}

Los ullales se caracterizan por una variedad bastante importante de copépodos, cuya variación estacional se representa en las Figs. 1 y 3, mostrando picos de abundacia limitados a determinadasépocas del año. Acontinuación se destacan las principales características de cada una de las especies de ciclópidos encontrados:

Metacyclops planus: Dussart, 1969, la describe como especie rara, de charcas más o menos temporales de la región circum-mediterránea de clima estepárico.En Españase conoce una única cita en Andalucía, en la laguna del Charro (Alonso, 1985).En nuestro estudio resultó ser el ciclópido planctónico más importante en el ullal de la Baldovina, con una poblacióndeadultosen el mesde junio.

Microcyclops rubellus major: Especie descrita como de aguas más bien distróficas, estival, sensible a la salinidad alta y capaz de resistir la desecación por aletargamiento (Dussart, 1969). Noha sidocitada en España con anterioridad yde este génerosólohay una cita Microcyclops varicans en los "aguamolls" de Tordera, de régimen temporal y salinidad baja (Alonso, 1985).M. rubellus se encuentra en el ullal Gros en primavera y verano, con un máximo muy marcado en agosto.

Acanthocyclops vernalis-robustus: Es una especie cosmopolita que se encuentra ampliamente distribuida por todo el país. Se encontró en los 


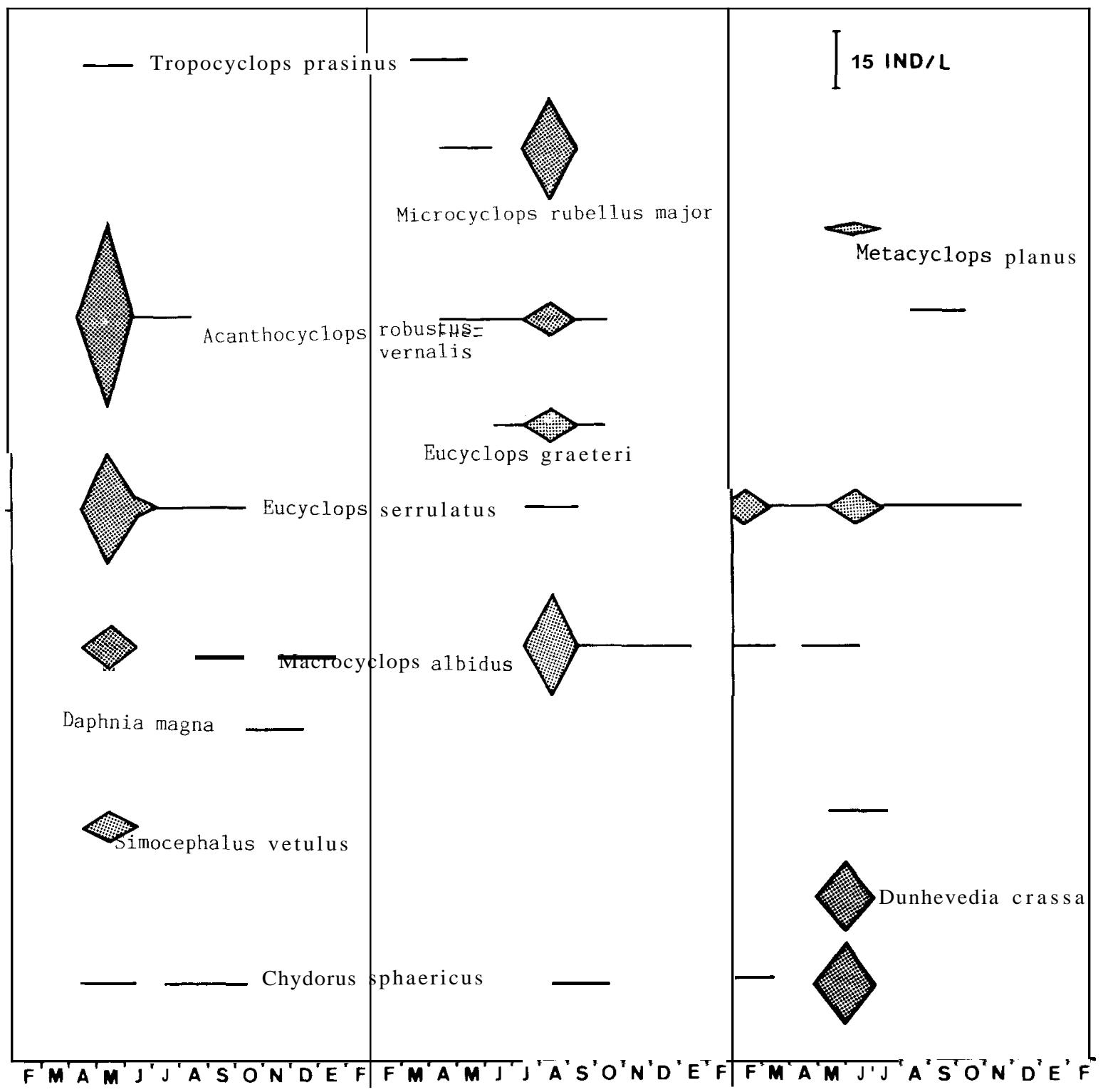

Figura 1.- Variación estacionalde los ciclópidos y clad6cerosen los tres ullales estudiados, desde febrero de 1986 a febrero de 1987 (meces en abcisas).

Seasonal variation of the cyclopoids and cladocers found in the three studied spring pools from February 1986 to Febmary 1987 (months in abscissas).

tres ullales, apareciendo como dominante en el ullal del Romanídurante el mes de mayo (Fig.1), siendo poco abundante en los otros. La especieo forma A.robusfuses la másfrecuente en los ullales del Romaní y Gros, mientras que en la Baldovina loes la forma A. vernalis setiger (Dussart 1969).A.. robustus es el zooplanctonte dominante en la Albufera. 
Eucyclops graeteri: Es una especie cavernícola que también se encuentra en aguas subterráneas; según Dussart (1969)ha sido hallada en la mitad hiporreica entre 4 y $6,5 \mathrm{~m}$ de profundidad. Según nuestros datos, sólo se ha encontrado en Francia y Centroeuropa; es pues primera cita para España, y de todas las muestras estudiadas, sólo se ha encontrado en el ullal Gros en verano, con un máximo en agosto (Fig. 1).

Eucyclopsserrula tus: Descrita por Alonso(1985), como especie que vive en el litoral de grandes lagos, lagunas y aguas temporales. Según este autor, es más frecuente en las aguas claras provistas de vegetación abundante, siendo una de las especies más eurioicas y eurícoras que se conocen. Su distribución es regular en todo el país, pero nunca ha sido citada con anterioridad en el lago de la Albufera. Se ha encontrado en los tres ullales, siendo abundante en el del Romaníy en el de la Baldovina.

Macrocyclops albidus: Descrita por Alonso (1985), como especie colonizadora del litoral de lagos y lagunas permanentes, y en ocasiones también de aguas temporales. Propia de aguas limpias con abundante vegetacióny pocomineralizadas. Esta especie fue encontrada en la Albufera por Blanco (1976), sin embargo en los estudios más recientessobre la Albufera,realizadosa partir del año 1980, no se ha encontrado. Se ha observado en los tres ullales.

Aunque de carácter más bentónico, varios harpactiocoides fueron también encontrados en el plancton de los ullales, a saber: Phyllognathopus viguieri y Nitocra spinipes en el ullal Gros, Horsiella brevicornis y Clefocarnptus retrogressus en el ullal de la Baldovina y Onychocarnptus mohammed en los ullales del Romaní y Gros.

De entre los cladóceros encontrados en los ullales (Fig. 1) destaca Dunhevedia crassa, la única especie del género que se encuentra en Europa; las otras especies son del Hemisferio Sur. En España, es particularmente frecuente en el Bajo Guadalquivir (Alonso, 1985), pero muy restringida en el restod e la Península. En esteestudio, sólo se encontró en la Baldovina durante el mes de junio.
En los ullales se observó la presencia de ostracodos y en el de la Baldovina se encontraron también algunos anfípodos y el decápodo $D$ ugastella valenfina, endémico de la zona levantina.

\section{Rotíferos}

Los rotíferos $\boldsymbol{s} \boldsymbol{e}$ caracterizaron por presentar una variabilidad muy grande. En la figura 2 se representa la dinámica de poblacionesdelasespecies más abundantes encontradas en los ullales.

Los géneros Brachionus y Lecane son los que presentan una mayor cantidad de especies en los ullales, con 9 y 13 especies respectivamente. Es interesante destacar la presencia de dos especies de Asplanchnopus, A. multiceps extremadamente rara en Europa y A. hyalinus propia de marjales salobres, también poco corriente, que se encuentra sólo en el ullal más salino de la Baldovina. Asplanchnopus en los ullales depredaba característicamente sobre Lecane quadridentata cuyos máximos fueron coincidentes en el ullal de la Baldovina. Los L. quadridentata fueron vistos repetidamente en el interior delos Asplanchnopus, bien directamente por transparencia o por adición de lejía diluida. También se vioen el interior de estos rotíferos alguna especie de Brachionus.

Una relación de las 47 especiesencontradas se muestra en la tabla $\mathbf{1}$, junto con los datos correspondientes de presencia, frecuencia relativa con respecto al total de individuos del zooplancton y abundancia máxima en cada uno de los ullales, así como una indicación de si han sido citadas en alguna época en la Albufera. El númerode especies comunes en los tres ullales fue 14, siendo el ullal de la Baldovina el más diversificado en cuanto a la composición específica de rotíferos, con 34 especies.

\section{Variación estaciona1 y diversidad}

En las figuras 1 y 2 se presentan las abundanciasa lolargodel tiempodelasespeciesdominantes en los tres ullales. Se observa cómo las máximas abundacias de las distintas especies coinci- 


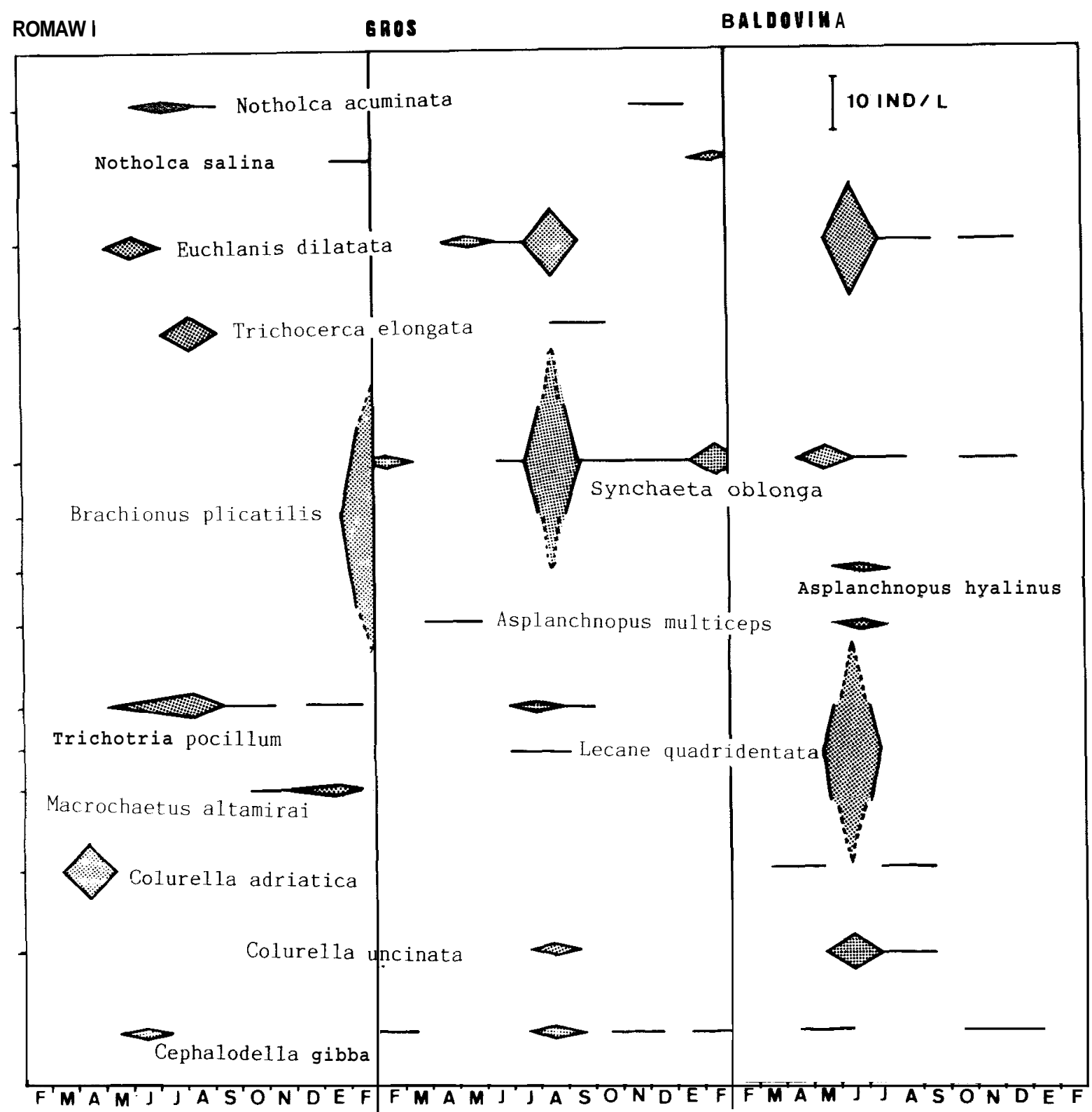

Figura 2.-Variaciónestaciona1 de las principalesespeciesde rotíferos en los tres ullales estudiados,desde febrerode 1986a febrero de 1987 (mesesen abcisas).

Seasonal variation of the mainspeciesof rotifers in the threestudied spring pools from February 1986 to February 1987 (months in abscissas).

den en el tiempo, disminuyendo su proporción considerablemente en el resto de los meses; sin embargo estos picos de abundancia se producen en diferentes épocas en cada ullal. En el Ullal del Romaníse produce en mayo, en el Gros en agosto y en el de la Baldovina en junio, locual señala una dinámica de poblaciones distinta para cada uno de ellos. Además presentan una composición específica diferente, caracterizado cada uno de ellos por una especie distinta de ciclópido. 
Tabla I.- Relacióndelas especies de rotíferosconindicación, para cada uno de los ullales, deln de muestras (Oc) en que la especie estaba presente (de un total de 12 muestrasen cada ullal), su frecuencia ( $F r$ ) en tantos por mil respecto del número total de individuos delzooplancton y la densidad máxima (Am) alcanzada, en ind $/ 1$, junto con la abreviatura del mes en que se registró dicho máximo (todos los meses citados corresponden a 1986, excepto En y Fb que corresponden a 1987). Se indica además con un (+) la presencia de la especie en la Albufera de Valencia registrada en muestras tomadas durante el primer y último cuarto de siglo.

\begin{tabular}{|c|c|c|c|c|c|c|c|c|c|c|c|}
\hline & & ROM & ANI & & GRO & & & ALDOV & INA & ALBU & FERA \\
\hline & Oc & $\%$ Fr & Am & Oc & $\% \circ \mathrm{Fl}_{1}$ & $\mathrm{r}$ Am & Oc & $\% \circ \mathrm{Fr}$ & Am & $\begin{array}{c}\text { (1) } \\
1918-29\end{array}$ & $\begin{array}{c}\text { (2) } \\
1974-82\end{array}$ \\
\hline Brachionus: B. plicatilis & 1 & 220,0 & $208 \mathrm{Fb}$ & 1 & 26,2 & $18 \mathrm{Fb}$ & 1 & 4,9 & $2 \mathrm{Fb}$ & & + \\
\hline B. urceolaris & & & & 1 & 0,1 & $1 \mathrm{My}$ & 1 & 0,2 & $1 \mathrm{My}$ & + & + \\
\hline B. bidentata & & & & & & & 1 & 0,6 & $1 \mathrm{Jl}$ & + & + \\
\hline B. quadndentatus f. brevispinus & & & & & & . & 2 & 1,7 & $1 \mathrm{My}$ & + & + \\
\hline B.quadridentatus var. cluniorbicularis & . & & & & & . & 1 & 0,3 & $1 \mathrm{Jl}$ & + & + \\
\hline B. calycifloms f. calyciflorus & & & & & & . & 1 & 0,5 & $1 \mathrm{My}$ & + & + \\
\hline B. calyciflorus var. dorcas & & & & & & . & 1 & 0,2 & $1 \mathrm{En}$ & + & + \\
\hline B. calycifloms f. anuraeiformis & & & & & & . & 1 & 0,2 & $1 \mathrm{Sp}$ & + & + \\
\hline B. angularis & & & & & & & 1 & 0,5 & $1 \mathrm{Sp}$ & + & + \\
\hline Keratella: K. quadrata & 1 & 0,8 & $1 \mathrm{Oc}$ & 1 & 0,2 & $1 \mathrm{Oc}$ & 2 & 0,9 & $1 \mathrm{Oc}$ & + & + \\
\hline K. tropica & 1 & 0,4 & $1 \mathrm{Nv}$ & . & & & 1 & 0,4 & $1 \mathrm{En}$ & & + \\
\hline K. cochlearis & 1 & 0,4 & $1 \mathrm{Ab}$ & 1 & 0,3 & $1 \mathrm{My}$ & . & & & & + \\
\hline Notholca: N. acuminata & 3 & 7,4 & $2 \mathrm{Jl}$ & 1 & 0,6 & $1 \mathrm{Dc}$ & . & & & + & + \\
\hline N. salina & 1 & 1,1 & $1 \mathrm{Fb}$ & 1 & 2,9 & $2 \mathrm{Fb}$ & . & & & & + \\
\hline N. striata & & & & 1 & 0.2 & $1 \mathrm{My}$ & & & & & \\
\hline Synchaetaoblonga & 1 & 7,4 & $7 \mathrm{Ag}$ & 7 & 175,3 & $104 \mathrm{Ag}$ & 3 & 9,0 & $4 \mathrm{My}$ & & + \\
\hline Tnchocerca elongata & 1 & 0,4 & $1 \mathrm{Ag}$ & 3 & 0,8 & $1 \mathrm{jl}$ & 1 & 0,4 & $1 \mathrm{Ag}$ & & \\
\hline Asplanchnopus: A. multiceps & & & & 1 & 0,1 & $1 \mathrm{My}$ & 1 & 3,0 & $2 \mathrm{Jn}$ & & \\
\hline A. hyalinus & & & & & & & 1 & 3,0 & $2 \mathrm{Jn}$ & & \\
\hline Polyarthraplatyptera & & & & & & & 1 & 1,1 & $1 \mathrm{En}$ & + & + \\
\hline Testudinella patina var. dendradera & & & & 1 & 0,3 & $1 \mathrm{My}$ & . & & & + & + \\
\hline Cephalodella gibba & 1 & 1,6 & $2 \mathrm{Jn}$ & 4 & 4,6 & $3 \mathrm{Ag}$ & 3 & 1,0 & $1 \mathrm{My}$ & & + \\
\hline Euchlanis dapidula & & & & & & & 1 & $<0,1$ & $13 \mathrm{Jn}$ & & \\
\hline Euchlanisdilatata & 1 & 8,0 & $5 \mathrm{Jn}$ & 4 & 20,3 & $11 \mathrm{Ag}$ & 4 & 17,2 & $8 \mathrm{Jn}$ & + & + \\
\hline Colurella: C. uncinata f. bicuspidata & 4 & 14,2 & $9 \mathrm{Jn}$ & 1 & 3,9 & $3 \mathrm{Ag}$ & 2 & 12,2 & $6 \mathrm{Jn}$ & + & \\
\hline C. adriatica & 1 & 11,4 & $11 \mathrm{My}$ & . & & . & 2 & 1,3 & $1 \mathrm{Ag}$ & + & + \\
\hline Mytilina ventralis & & & & & & & 1 & 0,4 & 1 Jn & + & + \\
\hline Trichotria: T. pocillum & 6 & 12,0 & $6 \mathrm{Ag}$ & 2 & 4,6 & $3 \mathrm{Ag}$ & . & & & + & + \\
\hline T. tetractis & & & & 2 & 12,2 & $8 \mathrm{~J} 1$ & & & & & \\
\hline Lepadelia: L ovalis & 6 & 17,4 & $8 \mathrm{Jn}$ & 2 & 10,2 & $5 \mathrm{Ag}$ & 3 & 62,4 & $33 \mathrm{Jn}$ & + & + \\
\hline L. patella & & & 3 & 1 & 0,4 & $1 \mathrm{Jn}$ & 1 & 0,2 & $1 \mathrm{My}$ & + & + \\
\hline Squatinella rostrum & & & & & & . & 1 & 0,4 & $1 \mathrm{Ag}$ & & \\
\hline Macrochaetusaltarnirai & 3 & 4,9 & $3 \mathrm{En}$ & . & & & & & & + & \\
\hline Lecane: L. luna & 2 & 1,5 & $1 \mathrm{Jl}$ & 3 & 4,5 & $3 \mathrm{Ag}$ & 4 & 104,1 & $57 \mathrm{Jn}$ & + & + \\
\hline L. lunaris & 5 & 8,4 & $4 \mathrm{Jn}$ & 1 & 1,0 & $1 \mathrm{Ag}$ & 1 & 0,4 & $1 \mathrm{Dc}$ & + & + \\
\hline L. bulla & 2 & 6,8 & $6 \mathrm{Jl}$ & 2 & 24,8 & $16 \mathrm{Ag}$ & 2 & 1,8 & $1 \mathrm{Jl}$ & + & + \\
\hline L.closterocerca & 7 & 34,9 & $13 \mathrm{Jl}$ & 6 & 7,2 & $3 \mathrm{Ag}$ & 4 & 41,0 & $20 \mathrm{Jn}$ & + & + \\
\hline L. hamata & 3 & 2,6 & $2 \mathrm{~J} 1$ & 1 & 2,9 & $2 \mathrm{Ag}$ & 3 & 1,1 & $1 \mathrm{Jn}$ & + & + \\
\hline L.quadridentata & & & - & 1 & 15,6 & $11 \mathrm{Ag}$ & 2 & 328,9 & $184 \mathrm{Jn}$ & + & + \\
\hline L. hastata & & & . & 1 & 0,2 & 1 Jn & . & & & & \\
\hline L. ungulata & & & & 2 & 1,5 & $1 \mathrm{Ag}$ & . & & & & \\
\hline L. stenroosi & & & & 1 & 1,5 & $10 \mathrm{C}$ & . & & & & + \\
\hline L. aculeata & 2 & 1,2 & $10 \mathrm{c}$ & . & & & & & & & + \\
\hline L. furcata & 1 & 0,8 & $1 \mathrm{Ag}$ & . & & & . & & & & + \\
\hline L. papuana & & & & & & & 1 & 1,0 & $1 \|$ & & + \\
\hline L. pideis & & & & & & & 1 & 0,4 & $1 \mathrm{Ag}$ & & \\
\hline Scaridium longicaudum & 1 & 0,4 & $1 \mathrm{Ag}$ & 1 & 1,5 & $1 \mathrm{Jl}$ & 1 & 0,4 & $1 \mathrm{Ag}$ & & \\
\hline Monommata sp. & 1 & 0,4 & $1 \mathrm{Ag}$ & 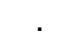 & & & 1 & 0,4 & $1 \mathrm{Jn}$ & & \\
\hline Bdelloidea & 8 & 11,4 & $2 \mathrm{Dc}$ & 11 & 58,4 & $34 \mathrm{Ag}$ & 8 & 6,4 & $2 \mathrm{Nv}$ & & \\
\hline
\end{tabular}

(1) Arevalo (1918) y Wiszniewski (1931); (2) Blanco (1976, 1981), Oltra y Miracle $(1981,1988)$ 
La densidad de poblaciónzooplanctónica (Fig. 4) sigue la variación estacional esperada con máximos en primavera en el ullaldel Romaní y el de la Baldovina. Sin embargo, en el ullal Gros la población zooplanctónica alcanzó su máximoen agosto, cuando en los otros ulalles se había ya iniciado el descenso de finales de verano-otoño. Este máximo tardío parece estar correlacionado con un aumento del fósforo en el agua y consiguiente crecimientodel fitoplancton, que se puede atribuir a la ocupación de las viviendas de recreo cercanas, en aquella época del año.

El máximode zooplancton correspondiente al mes de mayo en el ullal del Romanífue debido al crecimiento de las poblaciones de crustáceos, dominando las especies cosmopolitas de copépodos: Acanthocyclops vernalis-robusfus y Eucyclops serrulafus, mientras que los rotíferos presentaron un acusado máximo en febrero de 1987, constituido principalmente por Brachionus plicatilis (Tabla 1).

En el ullal Gros el zooplancton alcanzó su máxima densidad de población, tanto de crustáceos como de rotíferos en agosto, destacando entre los crustáceos las especies de distribución
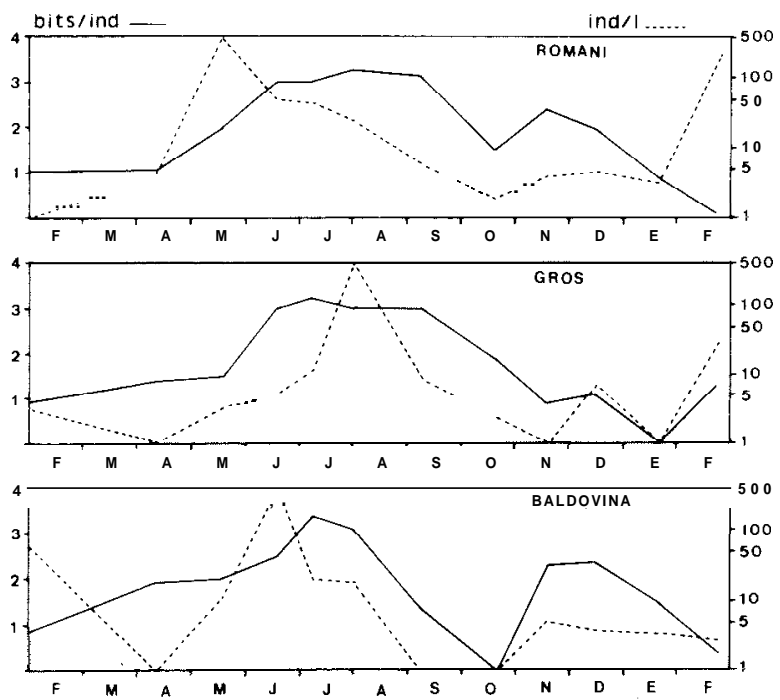

Figura 3.-Distribución estacionalde las proporciones relativas de cladóceros, ciclópidos y rotíferos en los tres uiiales desde febrero de 1986 a febrero de 1987.

Seasonal distribution of therelativeproportionsofcladocera, cyclopoids and rotifersin the threestudiedspring pools from February 1986 to Febniary 1987. cladocera $\square$ cyclopoida $\square$ rotifera

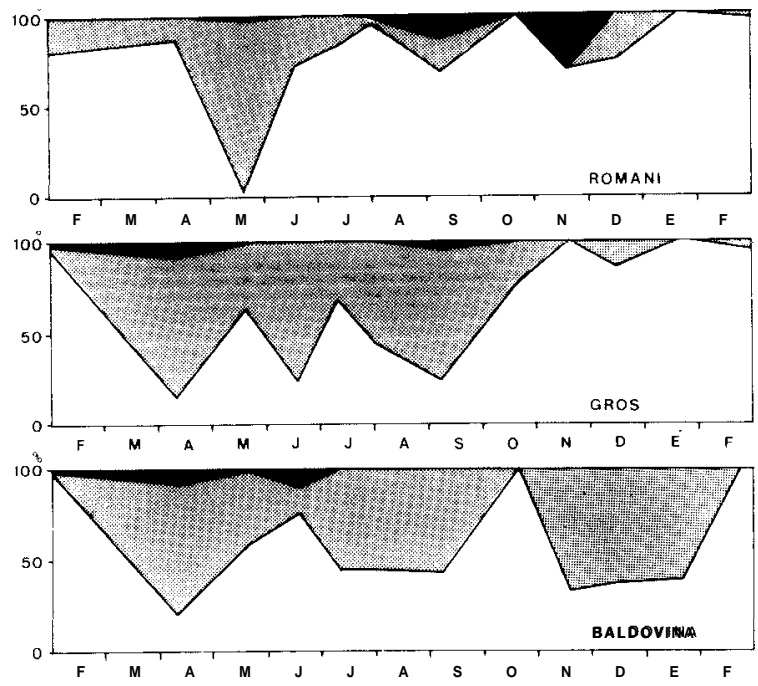

Figura 4.- Diversidad (bits / ind) y densidad zooplanctónica (ind/1) en los tres ullales desde febrero de 1986 a febrero de 1987.

Diversity and zooplanktonic population density in the three studied spring pools from February 1986 to February 1987.

restringida: Microcyclops rubellus y Eucyclops graeteri (nuevascitas en la Península Ibérica).En este mes fue también abundante el ciclópido Macrocyclopsalbidusy los rotíferos Euchlanisdilatata y Synchaeta oblonga.

En cambioen el ullal de la Baldovina los máximos de crustáceos y rotíferoscoincidieron en junio, peroeste máximode rotiferosfue debidoa especies litorales, principalmente del género Lecane (Lecane quadridentata fue la más abundante).Los crustáceos mayoritarios responsables del máximo en esta época, fueron tanto copépodos como cladóceros, más o menos en partes iguales. Debido en una buena proporción a especies raras de distribución restringida como Mefacyclops planus y Dunhevediacrassa, aunqueacompañadas de especies máscosmopolitas como Eucyclops serrulatus y Chydorus sphaericus.

Se ha calculado la diversidad de las comunidades zooplanctónicas constituidas por crustáceos y rotíferos, según el índice de ShannonWeaver, a lo largo del ciclo anual. Dicha diversidad, en los ullales, aumenta desde la primavera al otoño a medida queavanza la sucesión(Margalef, 
1982); este hecho queda reflejado en la figura 4, donde puede apreciarse que su valor máximose da entre junio y septiembre, según los distintos ullales, siendo mínima en primavera e invierno. Muestra también una tendencia a disminuir en octubre, siendo excepcionalmentebaja en el ullal de la Baldovina, producido seguramente por encontrarse el punto de muestreo demasiado cercano a la surgencia del mismo.

El número total de individuos, también representado en la Fig. 4, fue máximo en primavera, exceptoen el ullal Gros que fue en verano, debido al mencionado crecimiento tardío del fitoplancton. Como es de esperar los máximos de diversidad están desplazados con respecto a estos máximos de abundancia.

\section{DISCUSIÓN}

Los ullalesestudiados sonmanantiales de agua dulce, con poblacioneszooplanctónicasindependientes según las características de su enclave. Fundamentalmente los máximos de densidad zooplanctónica deben corresponder a máximos de producción primaria que tienen lugar en diferentes épocas en cada uno de los ullales.

Además las especies que componen el zooplancton son bastantes diferentesen cada uno de losullales.Cada uno puede caracterizarse por sus crustáceos planctónicos. El ullal del Romaníque presenta un grado de eutrofia más elevado, con aguas extraordinariamente ricas en nitratos, está dominado por Acanfhocyclops robusfus acompañadoen algunasépocas por cladóceros planctónicos. En elullal Gros, menoscontaminado, pueden vivir especies con mayores requerimientos como Microcyclops rubellus yademáscón hábitos subterráneos como Eucyclops graeferi. Finalmenteen el ullal de la Baldovina, de aguas algo más saladas que los dos anteriores, habita Mefacyclops planus, más tolerante que Microcyclops rubellus a la salinidad. Este ullal es además el que se encuentra en mejor estado, lo que se confirma por un número mayor de especies no cosmopolitas, tanto de crustáceos (comoel citado M. planus o Dunhevedia crassa) como de rotíferos (Tabla 1). En él se encontraron las dos especies mencionadas de
Asplanchnopus, de distribución restringiday Euchlanis dapidula, especie de la que hasta la fecha sólo existía una única cita mundial (Parise, 1966).

Asimismo los harpacticoides son claramente distintivos de los ullales Horsiella brevicornis y Cletocarnptus retrogressus harpacticoides eurialinos, tolerantes de salinidad se encuentran en el ullal de salinidades mayores y más fluctuantes, en el ullal del Romaní se encontró sólo una especie, la más cosmopolita Onychocampfusmohamed, mientras que en el ullal Gros se encontraron tres especies de harpacticoides, una de ellas típicamente habitante de aguas subterráneas en climas templados como Phyllognathopus viguieri.

La conexión con el zooplancton de la Albufera de Valencia se reduce en el caso de los crustáceos a las especies cosmopolitas Acamthocyclops robustus, Daphnia magna y Macrocyclops albidus (el 21\% de las especies).En cambio el $72 \%$ de las especies de rotíferosque se encuentran en algunos de los ullalesse han encontradoactualmenteen la Albufera, como se ve en la tabla 1, debido principalmente a su carácter colonizador; sin embargo, sólo un $25 \%$ de las especies han resultado comunes a los tres ullales, en nuestro estudio.

La existencia de especies de distribución restringida confirma la función de estos habitats comoáreas refugiode dichas especies y el in tcrés de su conservación.

\section{AGRADECIMIENTOS}

Se agradece la subvención del Institut Valencia d'Estudisi Investigacióotorgada a M. R. M. y el disfrute de una beca de la Conselleria de Cultura, Educació i Ciencia de la Generalitat Valenciana por uno de los autores (M. T. A.).

\section{Bibliografía}

Alonso, M. (1985): Lagunas españolas. Tesis doctoral. Fac. Biología, Barcelona, 795 pp.

Arévalo, C. (1918):Algunos rotíferos planctónicosde la Albufera de Valencia. Anal. Inst. Gral. Tec. Valencia, $\underline{8}:$ 1-47. 
Blanco, C. (1976): Estudio de la contaminación de la Albufera de Valencia y de los efectos de dicha contaminación sobre la fauna y flora del Lago. Tesis doctoral. Fac. Biología. Valencia, 193 pp.

Blanco, C. (1981):Evolución planctónica en una zona de eutrofia en regresión. Guaita $\underline{8}$ : 29-33.

Dussart, B. (1969):Les copépodes des eaux continentales d'Europe Occidentale. Vol. I. Boubée, París. 500 pp.

Margalef, R. (1982): Ecología. Omega. Barcelona. 951 pp.

Miracle, M. R.; Garcla, M. P. y Vicente, E. (1984): Heterogeneidad espacial de las comunidades fitoplanctónicas de la Albufera de Valencia. Limnética $\underline{1}: 20-31$.
Oltra, R. y Mikacle, M. R. (1984):Comunidades zooplanctónicas de la Albufera de Valencia. Limnéfica 1: 51-61.

Oltra, R. y MiRacle, M. R. (1988):Ciclo anual y diario de las poblaciones zooplanctónicas de la Albufera de Valencia. Archiv Hydrobiol (en prensa).

PARISE, A. (1966):The genus Euchlanis in the marsh of Fucuchio. Hydrobiologia 27:328-337.

SerRa, M.; Miracle, M. R. y Vicente, E. (1984):Interrelaciones entre los principales parámetros limnológicos de la Albufera de Valencia. Limnética 1:9-19. WisZNIEWSKI, J. (1931):Sur quelques rotifères trovés en Espagne. Arch. Hidrob. et Ichtiol. Varsovia 6: 1-41. 